\title{
Parâmetros nutricionais e bioquímicos de ovinos consumindo volumoso extrusado com di- ferentes teores de Uruchloa brizantha em comparação a silagem de milho tradicional
}

\author{
Carolina Moreira Araújo ${ }^{1 *}$; Karla Alves Oliveira²; Gilberto de Lima Macedo Junior ${ }^{3}$; Simone Pedro da Silva ${ }^{4}$; \\ Débora Adriana de Paula Silva ${ }^{5}$
}

DOI: https://doi.org/10.35699/2447-6218.2020.25810

\begin{abstract}
Resumo
Objetivou-se avaliar os parâmetros nutricionais, comportamento ingestivo e metabolismo energético e proteico de ovelhas alimentadas com diferentes tipos de volumoso extrusado. Vinte ovelhas Santa Inês, adultas, foram alocadas em gaiolas metabólicas individuais. O experimento teve duração de 21dias: quinze dias de adaptação e seis dias de coleta de dados. Os tratamentos foram dietas contendo silagem de milho ou volumoso extrusado com diferentes teores de Uruchloa brizantha em sua composição $(52,5 \%, 60 \%$ e $65 \%)$. Foi realizado ensaio para determinar o consumo de matéria seca (CMS) e digestibilidade aparente da matéria seca (DMS). A coleta de sangue para análise dos metabólitos sanguíneos foi realizada através de venopunção jugular. O comportamento ingestivo foi avaliado durante 24 horas. Utilizou-se o delineamento inteiramente ao acaso, com quatro tratamentos e cinco repetições. As médias dos tratamentos foram comparadas pelo teste de Tukey ao nível de $5 \%$ de significância. O CMS (kg/dia) e em relação ao peso corporal $(\mathrm{PC} \%)$ e o consumo de água e (litros/dia) foram superiores $(\mathrm{P}<0,05)$ nos animais que receberam volumoso extrusado. $\mathrm{O}$ peso das fezes apresentaram o mesmo padrão de resposta, e não alteraram o escore fecal. $\mathrm{O}$ tempo gasto em ingestão, ruminação e mastigação foram menores $(\mathrm{P}<0,05)$ para os animais que receberam exclusivamente silagem de milho, porém suas respectivas eficiências foram menores, quando comparadas com o volumoso extrusado. Os níveis de ureia sanguínea foram menores para os animais que receberam silagem de milho $(\mathrm{P}<0,05)$. Portanto, a utilização de volumoso extrusado com teor de Uruchloa brizantha de até $65 \%$ pode substituir a silagem de milho sem causar transtornos metabólicos para ovinos.
\end{abstract}

Palavras-chave: Extrusão. Fibra. Ovis aries.

\section{Nutritional and biochemical parameters of sheep consuming extruded roughage with different contents of Uruchloa brizantha compared to traditional corn silage}

\author{
Abstract

\footnotetext{
${ }^{1}$ Universidade Federal de Uberlândia. Uberlândia, MG. Brasil. https://orcid.org/0000-0003-4648-4971

${ }^{2}$ Universidade Estadual Paulista “Júlio de Mesquita Filho". Jaboticabal, SP. Brasil.

https://orcid.org/0000-0002-7792-2615

${ }^{3}$ Universidade Federal de Uberlândia. Uberlândia, MG. Brasil. https://orcid.org/0000-0001-5781-7917

${ }^{4}$ Universidade Federal de Uberlândia. Uberlândia, MG. Brasil. https://orcid.org/0000-0002-6391-1122

${ }^{5}$ Universidade Federal de Uberlândia. Uberlândia, MG. Brasil.

https://orcid.org/0000-0003-3052-0544

*Autor para correspondência: carolina.am@hotmail.com
}

The objective was to evaluate the nutritional parameters, feeding behavior and energy and protein metabolism of sheep fed with different types of extruded roughage. Twenty adult Santa Inês sheep were placed in individual metabolic cages. The experiment lasted 21 days: fifteen days of adaptation and six days of data collection. The treatments were diets containing corn silage or extruded roughage with different contents of Uruchloa brizantha in its composition $(52.5 \%, 60 \%$ and $65 \%)$. An experiment was carried out to determine dry matter intake (DMI) and apparent dry matter

Recebido para publicação em 13 de Outubro de 2020. Aceito para publicação 22 de Dezembro de 2020. e-ISSN: 2447-6218 / ISSN: 2447-6218. Atribuição CC BY. 
Araújo, C. M. et al.

digestibility (DMD). Blood collection for analysis of blood metabolites was performed through jugular venipuncture. Feeding behavior was assessed for 24 hours. A completely randomized design was used, with four treatments and five replications. The treatment means were compared using the Tukey test at a 5\% level of significance. The DMI (kg / day) and in relation to body weight (BW\%) and water intake (liters / day) were higher $(\mathrm{P}<0.05)$ in animals that received extruded roughage. The fecal weight showed the same response pattern, and did not change the fecal score. The time spent in ingestion, rumination and chewing were shorter $(P<0.05)$ for the animals that received exclusively corn silage, but their respective efficiencies were lower when compared to the extruded roughage. Blood urea levels were lower for animals that received corn silage $(\mathrm{P}<0.05)$. Therefore, the use of extruded roughage with an Uruchloa brizantha content of up to $65 \%$ can replace corn silage without causing metabolic disorders for sheep.

Key words: Extrusion. Fiber. Ovis aries.

\section{Introdução}

A fibra é o principal constituinte da dieta de ruminantes, pois é utilizada pelos microrganismos ruminais como principal fonte de energia (Mertens et al., 1994). A utilização de volumoso na composição da dieta de ruminantes é responsável por aumentar o custo de produção, uma vez que demanda investimentos em maquinários e mão-de-obra para sua produção, além de ser altamente dependentes de fatores climáticos, sendo que alguns erros podem comprometer a qualidade do alimento e reduzir o desempenho dos animais.

Uma alternativa para melhorar a digestibilidade da fibra do alimento, é a utilização de volumosos extrusados. $\mathrm{O}$ processo de extrusão caracteriza-se pelo cozimento em alta pressão (30 a $60 \mathrm{~atm}$ ), umidade (19 até $25 \%$ ) e temperatura ( 130 a $\left.140^{\circ} \mathrm{C}\right)$, em curto espaço de tempo (10 a 30 segundos) (Fagundes, 2005), o que promove alterações no alimento e provocam aumento na digestibilidade da fibra, amido e proteínas.

O processo de extrusão pode ainda melhorar a palatabilidade do alimento, o que associado à facilidade de apreensão do mesmo pode aumentar a eficiência de ingestão pelos animais (Andrigueto et al., 1981).

A falta ou excesso de nutrientes em uma dieta pode causar desbalanços no metabolismo dos animais devido ao desbalanço entre ingresso de nutrientes, sua metabolização e posterior excreção, podendo ocasionar possíveis quedas nos parâmetros nutricionais (Wittwer, 2000). O estudo do metabolismo animal surge como alternativa para evitar tais alterações, pois as mudanças são rapidamente detectadas.

Outra ferramenta que auxilia na avaliação nutricional dos ruminantes é o estudo do comportamento ingestivo. De acordo com Van Soest (1994), o tempo de ruminação é influenciado pela natureza da dieta, logo, o processo de extrusão pode ser uma alternativa para reduzir o tempo em ruminação.

Sendo assim, faz-se necessário a avaliação nutricional de volumosos extrusados a fim de se estabelecer como os mesmos devem atender as exigências dos animais e alcançar o máximo desempenho.
Objetivou-se avaliar os parâmetros nutricionais, comportamento ingestivo e metabolismo energético e proteico de ovelhas alimentadas com Uruchloa brizantha extrtusada em diferentes níveis de inclusão em comparação a silagem de milho

\section{Material e métodos}

O experimento foi realizado na Universidade Federal de Uberlândia, nos meses de fevereiro e março de 2017. Foram utilizadas vinte ovelhas da raça Santa Inês com mais de três anos e peso vivo inicial médio de $55 \mathrm{~kg}$, alocadas em gaiolas metabólicas individuais de piso ripado suspenso, contendo bebedouro, comedouro e cocho para sal mineral. $O$ experimento teve duração de 21 dias, sendo dividido em dois períodos: quinze dias de adaptação e seis dias destinados à coleta de dados.

Os tratamentos utilizados foram: silagem de milho (controle, forma convencional), Foragge ${ }^{\circledR} 52,5 \%$ (com 52,5\% de Uruchloa brizantha na forma extrusada), Foragge ${ }^{\circledR} 60,0 \%$ (com $60,0 \%$ de Uruchloa brizantha na forma extrusada) e Foragge ${ }^{\circledR} 65,0 \%$ (com $65,0 \%$ de Uruchloa brizantha na forma extrusada). Por se tratar de um produto comercial protegido por lei de patente segue apenas a informação dos constituintes do produto Foragge ${ }^{\circledR}$ (Composição: Uruchloa brizantha, amido, sal mineral e farelo de girassol). A alimentação foi fornecida duas vezes ao dia, as 8:00 e 16:00 horas. Os tratamentos foram ofertados como único alimento suprindo $3,5 \%$ do peso corporal inicialmente. Na tabela 1 tem a composição bromatológica da silagem de milho e do volumoso extrusado (Forrage ${ }^{\circledR}$ ) em diferentes níveis de inclusão da Uruchloa brizantha.

Para determinação do consumo alimentar, as sobras foram pesadas e sempre que os valores foram iguais à zero, aumentou-se a quantidade de alimento fornecido até atingir sobras equivalente a $10 \%$ do ofertado. As sobras de alimento foram colhidas e armazenadas em sacos plásticos devidamente identificados e mantidas em congelador a $-10^{\circ} \mathrm{C}$. Após o período de coleta as amostras eram homogeneizadas individualmente e uma alíquota representativa era separada para realização das análises bromatológicas. 
Parâmetros nutricionais e bioquímicos de ovinos consumindo volumoso extrusado com diferentes teores de Uruchloa brizantha em comparação a silagem de milho tradicional

Tabela 1 - Composição bromatológica da silagem de milho e do volumoso extrusado em diferentes níveis de inclusão da Uruchloa brizantha

\begin{tabular}{lcccccccc}
\hline Tratamento & $\begin{array}{c}\text { Uruchloa } \\
\text { brizantha }\end{array}$ & MS & PB & FDN & FDA & NDT & EE & Amido \\
\hline Silagem de milho & - & 31,20 & 8,00 & 51,11 & 36,17 & $59,66^{* *}$ & - & - \\
Foragge $^{\circledR} 52,5 \% *$ & 52,20 & 90,00 & 7,98 & 47,46 & 29,54 & 66,00 & 2,19 & 28,68 \\
Foragge $^{\circledR} 60,0 \% *$ & 60,00 & 91,20 & 7,67 & 50,33 & 33,38 & 65,81 & 1,66 & 24,34 \\
Foragge $^{\circledR} 65,0 \% *$ & 65,00 & 91,50 & 7,65 & 53,59 & 35,78 & 63,91 & 1,54 & 20,64 \\
\hline
\end{tabular}

MS = Matéria seca; $\mathrm{PB}=$ Proteína bruta; FDN = Fibra em detergente neutro; FDA = Fibra em detergente ácido; NDT = Nutrientes digestíveis totais; EE - Extrato etéreo; *Dados fornecidos pelo fabricante. *Teor de 52,5, 60 e 65\% de capim Uruchloa brizantha no produto Forrage ${ }^{\circledR}$ (Composição: Uruchloa brizantha, amido, sal mineral e farelo de girassol). ${ }^{* *} \mathrm{NDT}=87,84-(0,779 \mathrm{x} \% \mathrm{FDA})$ (Rodrigues, 2010).

O separador de partículas PennState ${ }^{\circledR}$ foi utilizado para quantificar o tamanho de partículas do alimento ofertado e das sobras de silagem. Os tamanhos das peneiras da PennState ${ }^{\circledR}$ são $>19 \mathrm{~mm}$ (peneira 1); 8 a 19 $\mathrm{mm}$ (peneira 2); 1,18 a $8 \mathrm{~mm}$ (peneira 3 ); $<1,18 \mathrm{~mm}$ (peneira fundo). Cada amostra de ofertado e sobra do alimento foi subdividida em quatro subamostras de 500 g. Essas subamostras foram colocadas na PennState ${ }^{\circledR}$ e para obter a separação das partículas foram realizados quatro movimentos "vai e vem" para cada lado da PennState, totalizando 16 movimentos para cada subamostra. Posteriormente obteve-se a média das repetições, possibilitando calcular a porcentagem de partículas retidas em cada peneira (Gráfico 1).

Gráfico 1 - Distribuição do tamanho das partículas de ofertado e sobras de silagem, utilizando o separador de partículas Penn State ${ }^{\circledR}$

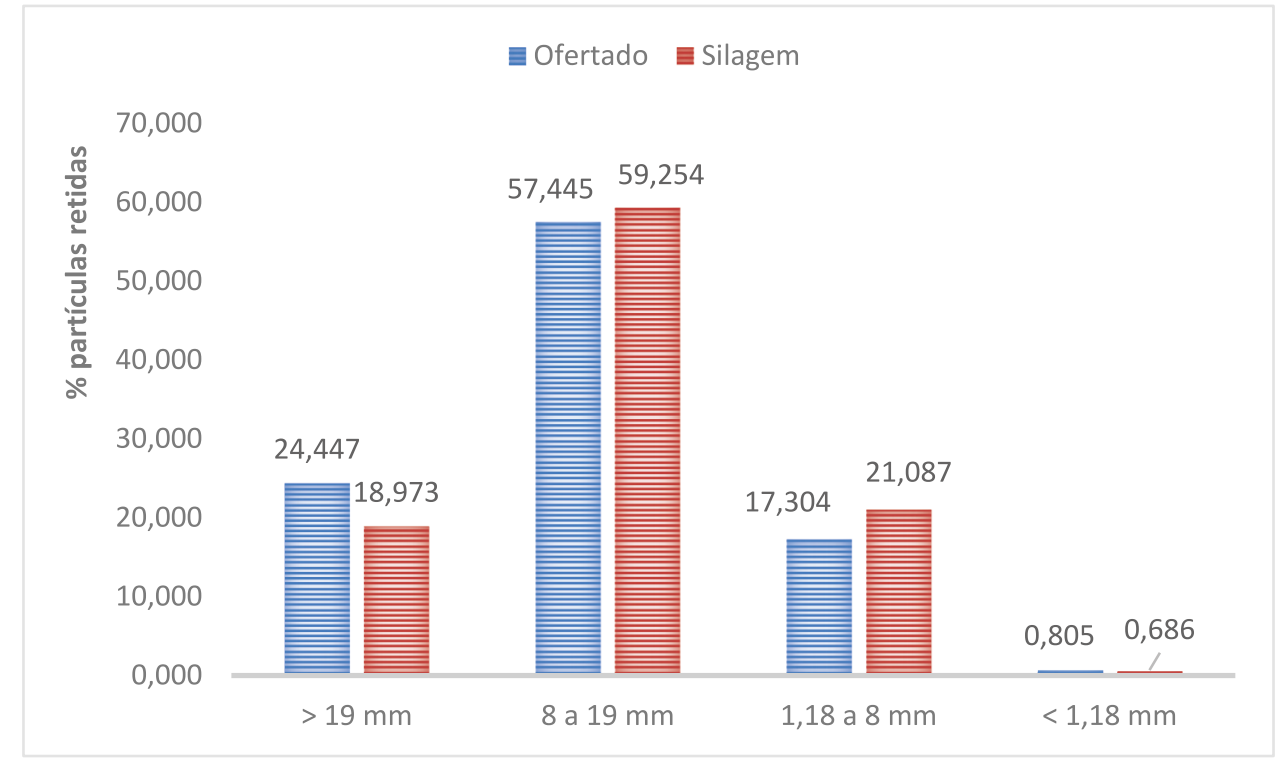

O cálculo do consumo de água oferecida no balde foi feito com base na diferença entre o ofertado (6 litros/animal) e as sobras, e, o cálculo do consumo de água total foi feito somando-se a água do balde com a água contida na ração ingerida. Ambos os cálculos foram feitos descontando a quantidade de água evaporada no dia, que foi obtida colocando-se 6 litros de água em um balde e subtraindo a sobra no dia seguinte. O balde utilizado para evaporação era igual aos utilizados como bebedouro pelos animais e alocado na mesma altura e próximo às gaiolas. Abaixo das gaiolas metabólicas era mantido artefato que permitia separação das fezes e unira, possibilitando avaliação da digestibilidade. Para realizar colheita total de fezes, as gaiolas eram limpas todos os dias pela manhã, com o auxílio de espátula e vassoura. As fezes foram pesadas, homogeneizadas e coletada alíquota representativa da amostra uma vez ao dia sempre no período da manhã. As alíquotas foram acondicionadas em sacos plásticos, identificados e mantidos em congelador a $-100 \mathrm{C}$.

Para avaliação do escore fecal utilizou-se a escala proposta por Gomes et al. (2012): 1- fezes ressecadas e sem brilho, 2- fezes normais, 3- fezes ligeiramente amolecidas, 4- fezes amolecidas, perdendo o formato e coladas umas às outras (cacho de uva), 5 - fezes amolecidas e sem formato normal, 6- fezes diarreicas. Era adicionado $100 \mathrm{~mL}$ de ácido sulfúrico $\left(\mathrm{H}_{2} \mathrm{SO}_{4}, 2 \mathrm{~N}\right)$ nos baldes coletoras de urina, evitando assim que houvesse perdas de nitrogênio por volatilização. $O$ volume de urina 
Araújo, C. M. et al.

foi mensurado diariamente pela manhã com o auxílio de proveta graduada (plástico). A densidade da urina foi avaliada no mesmo momento com o auxílio de refratômetro manual Megabrix ${ }^{\circledR}$. Foram retiradas alíquotas representativas deste material uma vez ao dia no período da manhã, e posteriormente foram acondicionadas em garrafas devidamente identificados e armazenadas em congelador a $-10^{\circ} \mathrm{C}$. Antes de serem armazenadas as amostras foram filtradas com filtro de papel (utilizados para coar café) retirando-se possíveis contaminações. Ao final do período de coleta, as amostras correspondentes a cada animal foram descongeladas, homogeneizadas e filtradas novamente utilizando-se filtro de papel, sendo retirada alíquota de $20 \%$ para posteriores análises laboratoriais. Posteriormente foi feita a primeira secagem das amostras de sobras e fezes em estufa de circulação forçada de ar, a $55^{\circ} \mathrm{C}$ por 72 horas, até obter peso constante. Feito isto, as amostras foram moídas, em moinho de facas do tipo Willey, em partículas de $1 \mathrm{~mm}$.

Logo após, as amostras foram levadas ao laboratório onde foi realizada a $2^{\underline{a}}$ matéria seca das amostras de sobras e fezes, em estufa a $105^{\circ} \mathrm{C}$ por 24 horas, sendo então possível calcular a matéria seca definitiva das mesmas e teor dos nutrientes, e posteriormente, a digestibilidade aparente dos nutrientes e matéria seca através das seguintes fórmulas (Maynard et al., 1984):

$$
\mathrm{CN}=(\text { Cons } \mathrm{x} \% \mathrm{Cons})-(\text { Sob } \mathrm{x} \% \mathrm{Sob}) ; \mathrm{DA}=(\mathrm{CN}-(\mathrm{Fez} \times \mathrm{Fez})) / \mathrm{CN} \times 100
$$

Onde: $\mathrm{CN}=$ consumo de nutriente $(\mathrm{kg})$; Cons = quantidade de alimento consumido (kg); \%Cons = teor de nutriente no alimento fornecido (\%); $\mathrm{Sob}=$ quantidade de sobra retirada $(\mathrm{kg}) ; \% \mathrm{Sob}=$ teor do nutriente nas sobras $(\%)$; DA = digestibilidade aparente $(\%)$; Fez = quantidade de fezes coletada $(\mathrm{kg}) ; \% \mathrm{Fez}=$ teor do nutriente nas fezes $(\%)$;

Para o estudo do comportamento ingestivo os animais foram submetidos à observação visual por pessoas treinadas, em sistema de revezamento, dispostas de maneira a não incomodar os animais, por um período de 24 horas, no último dia de coleta de dados. No período noturno, o ambiente recebeu iluminação artificial, e as luzes permaneceram acessas durante cinco dias antes da avaliação para promover a adaptação dos animais. Foram verificados a cada cinco minutos, se os animais estavam realizando ingestão do alimento e água, ruminação ou ócio, de acordo com a metodologia proposta por Fischer et al. (1998).

Os cálculos das atividades foram feitos em minutos por dia, admitindo que, nos cinco minutos subsequentes a cada observação, o animal permaneceu na mesma atividade. Já o tempo total gasto em mastigação (MAST) foi determinado somando-se os tempos gastos em ingestão (ING) e ruminação (RUM). As eficiência de ingestão (EIng), mastigação (EMast) e ruminação (ERum) foram obtidas segundo Polli, et al. 1996, de acordo com as equações: EIng $(\mathrm{g} / \mathrm{min})=\mathrm{CMS} / \mathrm{Tal}$; EMast $(\mathrm{g} / \mathrm{min})$ = CMS/Tmast e ERum $(\mathrm{g} / \mathrm{min})=\mathrm{CMS} /$ Trum; em que CMS é consumo de MS ( $\mathrm{g} / \mathrm{MS} / \mathrm{dia}$ ), Tal é o tempo de alimentação (h/dia), Tmast é o tempo em mastigação (h/ dia) e Trum é o tempo em ruminação (h/dia) (Bürger et al. 2000).

As colheitas de sangue para avaliação dos componentes bioquímicos foram realizadas por venopunção jugular com auxílio de tubos Vacutainer ${ }^{\circledR}$ sem anti-coagulante, durante três dias alternados (primeiro, terceiro e quinto dia de coleta de dados). Os dados apresentados foram obtidos através da média dos três dias de coleta. Todas as colheitas de sangue foram realizadas no período da manhã, antes do fornecimento da primeira alimentação. As amostras de sangue coletadas foram centrifugadas a $3000 \mathrm{rpm}$ por 10 minutos, sendo os soros separados em alíquotas, guardados em microtubos e armazenados em freezer a $-5^{\circ} \mathrm{C}$ para posterior análise laboratorial. Os componentes bioquímicos para determinação do metabolismo energético foram: triglicerídeos, colesterol e VLDL (lipoproteína de muito baixa densidade); e, para determinação do metabolismo proteico foram: proteína total, albumina, globulina, ácido úrico, ureia e creatinina.

A avaliação glicêmica foi feita no último dia de coleta, sendo que neste dia a primeira alimentação foi realizada logo após a primeira coleta de sangue e a segunda alimentação após a última coleta. As coletas de sangue foram realizadas às 8:00, 11:00, 14:00, 17:00 e 20:00 horas, sendo as amostras coletadas por venopunção jugular com auxílio de Vacutainer ${ }^{\circledR}$ com fluoreto. Todas as amostras foram processadas em analisador bioquímico automatizado (Bioplus ${ }^{\circledR}$ 2000), usando kit comercial da $\mathrm{GT} \mathrm{Lab}^{\circledR}$.

Utilizou-se o delineamento inteiramente ao acaso, com quatro tratamentos e cinco repetições por tratamento. As médias dos tratamentos foram comparadas pelo teste de SNK ao nível de 95\% de significância, utilizando o pacote estatístico SAEG 9.0. A glicemia foi em esquema de parcelas subdivididas, sendo tratamentos nas parcelas e horários (foram analisados por estudo de regressão) de colheita nas subparcelas. As médias de escore fecal foram avaliadas pelo teste não paramétrico de Kruskal e Wallis (1952). Para todas as variáveis foram testadas a normalidade e homogeinicidade dos dados.

\section{Resultados e discussão}

Os animais alimentados com volumoso extrusado contendo diferentes teores de Uruchloa brizantha (52,5\%, $60,0 \%$ E 65,0\%) tiveram maior consumo de matéria seca expresso em $\mathrm{kg} /$ dia e em relação ao peso corporal do que os animais alimentados com silagem de milho $(\mathrm{P} \leq 0,05$; Tabela 2). De acordo com o NRC (2007) o consumo de matéria seca ideal para ovelhas adultas em manutenção 
Parâmetros nutricionais e bioquímicos de ovinos consumindo volumoso extrusado com diferentes teores de Uruchloa brizantha em comparação a silagem de milho tradicional

é de $1,83 \%$ em relação ao peso corporal. Entretanto, no presente estudo, o consumo manteve-se acima do recomendado em todos os tratamentos.

Para o tratamento silagem o consumo foi $8,04 \%$ acima do recomendado, enquanto o tratamento extrusado $65,0 \%$ foi 59,33\% acima do recomendado. Esta diferença pode estar relacionada ao efeito de enchimento ruminal causado nos animais que consumiram exclusivamente silagem de milho. Ou seja, a silagem por conter partículas maiores permaneceu no rúmen por mais tempo (gráfico 1), sofrendo os efeitos físicos, provenientes da mastigação durante o processo de ruminação e da digestão pelos microrganismos ruminais. Como observado, 59,25\% do alimento ofertado continham partículas com tamanho entre 8 e 19 mm (Gráfico 1) o que contribuiu para este efeito.

Tabela 2 - Consumo e digestibilidade aparente da matéria seca em ovelhas alimentadas com diferentes níveis de Uruchloa brizantha no volumoso extrusado em comparação à silagem de milho.

\begin{tabular}{lccc}
\hline Tratamento & CMS (kg/dia) & CMS/PC (\%) & DMS (\%) \\
\hline Silagem de milho* $^{*}$ & $1,08 \mathrm{~B}$ & $1,99 \mathrm{~B}$ & 58,84 \\
Foragge $^{\circledR 52,5 \%}$ & $2,33 \mathrm{~A}$ & $3,70 \mathrm{~A}$ & 61,46 \\
Foragge $^{\circledR} 60,0 \%$ & $2,44 \mathrm{~A}$ & $4,37 \mathrm{~A}$ & 53,61 \\
Foragge $^{\circledR} 65,0 \%$ & $2,60 \mathrm{~A}$ & $4,50 \mathrm{~A}$ & 61,20 \\
\hline MG & $\mathbf{2 , 0 9}$ & $\mathbf{3 , 6 4}$ & $\mathbf{5 8 , 7 8}$ \\
CV & $\mathbf{1 8 , 9 0}$ & $\mathbf{1 7 , 1 0}$ & $\mathbf{1 0 , 6 1}$ \\
\hline
\end{tabular}

CMS = Consumo de matéria seca; PC = Peso corporal; DMS = Digestibilidade da matéria seca; MG = Média geral; CV = Coeficiente de variação; Letras distintas na coluna diferem-se pelo Teste de Tukey a 5\%. *Silagem de milho na forma convencional como tratamento controle.

Os primeiros estudos que comprovam isso foram conduzidos por Campling et al. (1961), quando observaram que quando haviam taxas semelhantes de matéria seca no retículo-rúmen de vacas o consumo passava a ser dependente da velocidade de escoamento deste material. O consumo observado por estes autores aumentou significativamente quando o alimento ingerido era retirado através de cânulas ruminais. $O$ tamanho e quantidade de fibra presente na dieta pode influenciar na formação do "match" ruminal, constituído por um emaranhado de partículas flutuantes do alimento, recém ingeridas e em processo de fermentação pelos microrganismos no rumen. Quanto maiores as partículas de alimento ingeridas, maior será a formação do "match" ruminal, retardando a taxa de passagem do alimento e consequentemente diminuindo o CMS (Gomes et al. 2012). O volumoso extrusado, por sua vez, possui partículas menores (2 $\mathrm{mm}$ ) e os nutrientes apresentarem-se mais facilmente digestíveis, pelo processo de extrusão, o que permitiu que os animais consumissem até que pudessem atingir sua demanda energética e física.

Apesar do maior consumo de matéria seca pelas ovelhas que receberam volumoso extrusado, não houve efeito sobre a digestibilidade da matéria seca ( $P>0,05 ;$ Tabela 2). Este resultado é interessante, visto que a fibra é responsável por auxiliar na manutenção da saúde ruminal. O processo de extrusão torna a fibra mais facilmente digestível, contribuindo para a obtenção destes resultados. Senger et al., (2005) encontraram valores para digestibilidade in vitro da matéria seca da silagem de milho variando de 46,2 a $57,9 \%$, valor este mais baixo do apresentado pelos volumosos extrusados do presente estudo que foi em média 58,75\%. Isso mostra que o volumoso extrusado usado nesse estudo pode ser mais eficiente quando comparado a silagem de milho, pois permitiu maior CMS sem afetar a digestibilidade e está ainda se manteve acima do observado para silagens convencionais na literatura como citado acima.

$\mathrm{O}$ consumo de água total $\left(\mathrm{CH}_{2} \mathrm{O}\right)$ foi maior para os animais alimentados com volumoso extrusado ( $P>0,05$; Tabela 3) em comparação aos que receberam silagem de milho, o que se deve ao teor de matéria seca dos volumosos, sendo que a silagem de milho apresenta $31,20 \%$ de MS enquanto o volumoso extrusado variou entre 88,43 e $91,00 \%$ de MS. De acordo com a equação proposta por Forbes (1968) que possibilita calcular o requerimento de ingestão de água diária a partir do CMS através da fórmula: $\mathrm{CH}_{2} \mathrm{O}=3,86$ x CMS - 0,99, temos que a recomendação de ingestão de água pelos animais que foram alimentados com silagem de milho é de 3,18 1/dia enquanto para os alimentados com volumoso extrusado é de $8,46 \mathrm{l} /$ dia. Nota-se que a volume de água ingerida pelos animais ficou 67 e $51 \%$ abaixo do valor recomendado para aqueles que consumiram silagem e volumoso extrusado, respectivamente (Tabela 3).

O volume e a densidade da urina não foram influenciados pelos tipos de volumosos estudados $(\mathrm{P}<0,05$; Tabela 3). Indicando que de fato, o maior consumo de água pelos animais recebendo volumoso extrusado foi para suprir sua falta no alimento. Segundo Reece (2006), a excreção de urina deve ser entre $100-400 \mathrm{~mL}$ para cada $10 \mathrm{~kg}$ de peso corporal em ovinos. Sendo assim, a excreção média de $0,524 \mathrm{~mL}$ de urina observada no presente 
Araújo, C. M. et al.

estudo manteve-se dentro da normalidade. A média de densidade da urina manteve-se $1,7 \%$ acima do proposto por Hendrix (2005), que sugere uma variação de 1,020 a 1,040 g/dL. Mesmo com a baixa ingestão de água os animais não apresentaram queda no CMS e DMS (Tabela 2), sendo assim, podemos concluir que os mesmos não sofreram estresse hídrico, uma vez que este é o maior limitante para o CMS (Araújo Filho, 2006).

Houve efeito do tipo de volumoso sobre o peso das fezes na matéria natural e na matéria seca $(\mathrm{P}<0,05$; Tabela 3). As ovelhas alimentadas com volumoso extrusado contendo 60 e $65 \%$ de Uruchloa brizantha tiveram maior peso das fezes na matéria natural. Já para o peso das fezes na matéria seca, valores superiores foram observados em todos os tratamentos com volumoso extrusado e inferior para silagem de milho (o valor médio do peso das fezes na matéria seca dos tratamentos com Uruchloa brizantha em diferentes níveis de inclusão foi $111 \%$ maior em comparação ao de silagem de milho). Maior peso de fezes é resultado do maior consumo (Tabela 2), entretanto, não houve queda da digestibilidade e o escore fecal manteve-se dentro dos valores considerados normais. Segundo Gomes et al., (2012) o escore ideal seria 2, e é um indicativo da condição de saúde dos animais dentro da normalidade.

Tabela 3 - Consumo de água total $\left(\mathrm{CH}_{2} \mathrm{O}\right)$, consumo de água em relação ao consumo de matéria seca $\left(\mathrm{CH} \mathrm{H}_{2} \mathrm{O} / \mathrm{CMS}\right)$, volume urinário (Vol. Ur.), densidade urinária (Dens. Ur.), produção fecal expresso na matéria natural (PFMN), expresso na matéria seca (PFMS), matéria seca das fezes (MSF) e escore fecal (EF) de ovelhas alimentadas com diferentes níveis de Uruchloa brizantha no volumoso extrusado em comparação à silagem de milho

\begin{tabular}{lcccc}
\hline Tratamento & $\mathrm{CH}_{2} \mathbf{O}(\mathrm{L})$ & $\mathrm{CH}_{2} \mathrm{O} / \mathrm{CMS}(\mathrm{L} / \mathbf{k g})$ & Vol. Ur. (L) & Dens. Ur. \\
\hline Silagem de milho* & $1,05 \mathrm{~B}$ & 1,86 & 0,805 & 1,0262 \\
Foragge $^{\circledR} 52,5 \%$ & $4,00 \mathrm{~A}$ & 1,65 & 0,408 & 1,0454 \\
Foragge $^{\circledR} 60,0 \%$ & $4,49 \mathrm{~A}$ & 1,82 & 0,407 & 1,0424 \\
Foragge $^{\circledR} 65,0 \%$ & $3,87 \mathrm{~A}$ & 1,75 & 0,478 & 1,1186 \\
\hline MG & $\mathbf{3 , 3 5}$ & $\mathbf{1 , 7 7}$ & $\mathbf{0 , 5 2 4}$ & $\mathbf{1 , 0 5 8 1}$ \\
CV & $\mathbf{3 3 , 1 9}$ & $\mathbf{1 2 , 4 1}$ & $\mathbf{3 8 , 5 6}$ & $\mathbf{8 , 5 6}$ \\
\hline Tratamento & PFMN (kg) & PFMS (g) & MSF (\%) & $\mathbf{E F *}$ \\
\hline Silagem de milho* & $1,40 \mathrm{~B}$ & $0,510 \mathrm{~B}$ & 38,03 & 2,16 \\
Foragge $^{\circledR 52,5 \%}$ & $2,32 \mathrm{AB}$ & $0,952 \mathrm{~A}$ & 40,84 & 3,04 \\
Foragge $^{\circledR} 60,0 \%$ & $3,34 \mathrm{~A}$ & $1,104 \mathrm{~A}$ & 35,70 & 2,76 \\
Foragge $^{\circledR} 65,0 \%$ & $2,84 \mathrm{~A}$ & $1,178 \mathrm{~A}$ & 40,98 & 2,64 \\
\hline MG & $\mathbf{2 , 4 7}$ & $\mathbf{0 , 9 3 6}$ & $\mathbf{3 8 , 8 9}$ & $\mathbf{2 , 6 5}$ \\
CV & $\mathbf{3 2 , 3 1}$ & $\mathbf{3 4 , 1 3}$ & $\mathbf{2 8 , 9 7}$ & $\mathbf{x x x}$ \\
\hline
\end{tabular}

MG = Média geral; CV = Coeficiente de variação; *Estatística não paramétrica; Letras distintas na coluna diferem-se pelo Teste de Tukey a 5\%.

*Silagem de milho na forma convencional como tratamento controle.

A matéria seca fecal apresentou média de 38,89\% (Tabela 3), estando dentro do recomendado para a espécie segundo Van Clef et al. (2010) que é de 37 a 44\%. As diferenças citadas acima estão diretamente relacionadas com o aumento no CMS pelos animais que consumiram volumoso extrusado, e possivelmente maior tempo de retenção da digesta no rúmen pelos animais que consumiram exclusivamente silagem, devido ao maior tamanho de partícula deste alimento (Gráfico 1 ).

Todas as variáveis analisadas no comportamento ingestivo dos animais apresentaram diferença significativa $(\mathrm{P} \leq 0,05)$ entre os tratamentos (Tabela 4). As ovelhas que receberam silagem de milho como volumoso permaneceram maior tempo em ingestão. Menor tempo em ingestão foi gasto pelos animais que receberam volumoso extrusado com 52,5\% de inclusão de capim-braquiária (Tabela 4), aumentando simultaneamente com o aumento na porcentagem de fibra no volumoso extrusado. $\mathrm{O}$ volumoso extrusado é mais facilmente apreendido pelos animais quando comparado à silagem de milho, o que leva ao menor tempo gasto em ingestão dos alimentos extrusados. Concomitantemente, a eficiência de ingestão (Ef. Ing.) foi maior para os animais que receberam ração extrusada, independente do nível de inclusão de fibra, o que não ocorre em dietas contendo volumosos convencionais, pois estes requerem maior tempo para a manipulação do alimento pelo animal, e para a digestão pelos microrganismos ruminais. Novamente, as mudanças que ocorrem nos nutrientes durante o processo de 
Parâmetros nutricionais e bioquímicos de ovinos consumindo volumoso extrusado com diferentes teores de Uruchloa brizantha em comparação a silagem de milho tradicional

extrusão (gelatinização do amido, ruptura das ligações entre carboidratos e proteínas) contribuíram para estes resultados, além da diferença no tamanho das partículas. Vale ressaltar que o tratamento com $65 \%$ de Uruchloa apresentou tempo em ingestão igual ao tratamento contendo apenas silagem, mostrando que a maior inclusão de fibra no volumoso extrusado o deixou com características semelhantes à silagem. Isso mostra que além do tama- nho da partícula do alimento, outro fator que interfere no tempo de ingestão é a porcentagem de fibra, pois o tratamento com 65\% de Uruchloa continha maior teor de FND quando comparados aos demais (Tabela 1). A fato dos animais alimentados com 65\% de Uruchloa extrusada terem apresentado maior CSM e CMS/PC (Tabela 2) também pode ter contribuído para o maior tempo em ingestão.

Tabela 4 - Comportamento ingestivo (minutos/dia) e consumo de matéria seca em relação ao tempo de ingestão, ruminação e mastigação total $(\mathrm{g} / \mathrm{min})$ em ovelhas alimentadas com diferentes níveis de Uruchloa brizantha no volumoso extrusado em comparação à silagem de milho.

\begin{tabular}{|c|c|c|c|c|}
\hline Tratamento & ING (min) & RUM (min) & ÓCIO (min) & MAST (min) \\
\hline Silagem de milho* & $267,0 \mathrm{~A}$ & $396,0 \mathrm{~A}$ & 777,0 B & $663,0 \mathrm{~A}$ \\
\hline Foragge ${ }^{\circledR} 52,5 \%$ & $147,0 \mathrm{C}$ & $185,0 \mathrm{~B}$ & $1.108,0 \mathrm{~A}$ & $332,0 \mathrm{~B}$ \\
\hline Foragge ${ }^{\circledR} 60,0 \%$ & $169,0 \mathrm{BC}$ & $179,0 \mathrm{~B}$ & $1.092,0 \mathrm{~A}$ & $348,0 \mathrm{~B}$ \\
\hline Foragge $^{\circledR} 65,0 \%$ & $232,0 \mathrm{AB}$ & $166,0 \mathrm{~B}$ & $1.042,0 \mathrm{~A}$ & $398,0 \mathrm{~B}$ \\
\hline MG & 203,75 & 231,50 & $1.004,75$ & 435,25 \\
\hline CV & 27,74 & 32,22 & 10,73 & 24,76 \\
\hline Tratamento & E.Ing ( $\mathrm{g} / \mathrm{min})$ & E.Rum $(\mathrm{g} / \mathrm{min})$ & E.Mast (g/min) & \\
\hline Silagem de milho* & $4,05 \mathrm{~B}$ & $2,81 \mathrm{~B}$ & $1,65 \mathrm{~B}$ & \\
\hline Foragge $^{\circledR} 52,5 \%$ & $15,87 \mathrm{~A}$ & $13,33 \mathrm{~A}$ & $6,84 \mathrm{~A}$ & \\
\hline Foragge ${ }^{\circledR} 60,0 \%$ & $15,38 \mathrm{~A}$ & $13,90 \mathrm{~A}$ & 7,09 A & \\
\hline Foragge ${ }^{\circledR} 65,0 \%$ & $12,12 \mathrm{~A}$ & $18,41 \mathrm{~A}$ & $7,06 \mathrm{~A}$ & \\
\hline MG & 11,85 & 12,11 & 5,66 & \\
\hline CV & 28,73 & 40,68 & 27,37 & \\
\hline
\end{tabular}

Ing. = Ingestão; Rum. = Ruminação; Mast. = Mastigação; E. = Eficiência; MG = Média geral; CV = Coeficiente de variação. Médias seguidas de letras distintas na coluna diferem entre si pelo teste de Tukey. *Silagem de milho na forma convencional como tratamento controle.

O tempo gasto para ruminação (minutos/dia) foi maior nos animais que receberam silagem de milho $(\mathrm{P}<0,05$; Tabela 4). Isso ocorre porque no volumoso extrusado os nutrientes são altamente digestíveis e possuem partículas menores ( $2 \mathrm{~mm})$, levando a um menor tempo em ruminação, como verificado por Gomes et al. (2012). Ao contrário do volumoso extrusado, a silagem de milho possui partículas maiores, entre 8 e $19 \mathrm{~mm}$ (Gráfico 1), e os nutrientes são menos digestíveis e a taxa de passagem do alimento é reduzida, sendo assim é necessário maior tempo em ruminação para que estas partículas atinjam tamanhos desejados e possam seguir para os próximos compartimentos digestórios, indicando a possível formação do match ruminal. Isso também explica o aumento no consumo de MS (Tabela 2) para os animais alimentados com volumoso extrusado. Esse aumento no CMS está relacionado ao menor tempo gasto em ruminação, indicando maior taxa de passagem do alimento pelo trato gastrointestinal e consequente esvaziamento do rúmen. Mesmo assim, a digestibilidade não foi afetada (Tabela 2) indicando que o tempo gasto em ruminação foi suficiente para suprir as necessidades fisiológicas dos animais. Menor tempo em ruminação pode diminuir a produção de saliva e causar desequilíbrios na manutenção do $\mathrm{pH}$ ruminal. Mesmo havendo diferença de tempo de ruminação de 3 horas e 31 minutos entre o tratamento silagem e o extrusado, nenhum distúrbio ruminal foi observado. Esta diferença está também relacionada aos maiores tamanhos das partículas da silagem de milho (Gráfico 1).

O tempo gasto em mastigação foi menor $(\mathrm{P}<0,05)$ para os animais que receberam volumoso extrusado (Tabela 4). O processo de mastigação pelos ruminantes é fundamental para a manutenção da saúde ruminal, tendo em vista que durante a mastigação ocorre a produção da saliva que é rica em íons fosfato e bicarbonato que são agentes tamponantes e que evitam quedas no $\mathrm{pH}$ do rúmen, o que reduz o desenvolvimento de problemas, como por exemplo, acidose. Ressalta-se que tais problemas não foram vistos nesse experimento como citado anteriormente. 
Araújo, C. M. et al.

A eficiência de ruminação foi menor para o tratamento silagem de milho. O tempo de permanência em ócio foi maior para os animais que receberam volumoso extrusado ( $\mathrm{P}<0,05$; Tabela 4). Como estes animais passaram menor tempo ingerindo e ruminando, consequentemente permaneceram maior parte do dia em ócio, comparado com aqueles que receberam silagem de milho, o que aumenta a probabilidade de elevar o CMS, como observado na Tabela 2 . A diferença de tempo de permanência em ócio entre os tratamentos silagem de milho e volumoso extrusado foi de aproximadamente cinco horas. Maior tempo em ócio pode garantir aos animais menor gasto energético para desempenhar outras funções, proporcionando possíveis ganhos no seu desempenho. Os parâmetros avaliados para o comportamento ingestivo foram influenciados de forma positiva pela inclusão de volumoso extrusado até $65 \%$, não alterando de forma negativa o comportamento ingestivo dos animais.

As ovelhas que receberam volumoso extrusado com maiores teores de Uruchloa brizantha (60 e 65\%) tiveram as maiores concentrações de ureia sanguínea ( $<<0,05$; Tabela 5), enquanto os animais alimentados exclusivamente com silagem de milho tiveram as menores concentrações. Segundo González e Scheffer (2002) a ureia é indicador sensível e imediato da ingestão de proteína e é sintetizada em quantidades proporcionais a concentração de amônia produzida no rúmen, sendo esta tóxica para ruminantes quando presente em altas concentrações. A menor concentração de ureia sanguínea observada nos animais que receberam exclusivamente silagem pode indicar melhor uso do nitrogênio no rúmen. Enquanto, as maiores concentrações obtidas podem indicar falta de sincronismo entre os carboidratos e nitrogênio no ambiente ruminal, refletindo em picos de ureia. Além disso, podemos observar que os níveis de amido diminuíram à medida em que houve aumento na inclusão de fibra no volumoso extrusado (Tabela 1), indicando que pode ter ocorrido falta de esqueleto de carbono de rápida degradação ruminal.

Tabela 5 - Metabolitos proteicos e energéticos de ovelhas alimentadas com diferentes níveis de Uruchloa brizantha no volumoso extrusado em comparação à silagem de milho.

\begin{tabular}{ccccc}
\hline Tratamento & $\begin{array}{c}\text { Creatinina } \\
(\mathbf{m g} / \mathrm{dL})\end{array}$ & $\begin{array}{c}\text { Ureia } \\
(\mathbf{m g} / \mathrm{dL})\end{array}$ & $\begin{array}{c}\text { Ácido úrico } \\
(\mathbf{m g} / \mathrm{dL})\end{array}$ & $\begin{array}{c}\text { Albumina } \\
(\mathrm{g} / \mathrm{dL})\end{array}$ \\
\hline Silagem de milho* & 0,86 & $16,6 \mathrm{C}$ & 0,16 & 3,49 \\
Foragge $^{\circledR} 52,5 \%$ & 0,77 & $21,53 \mathrm{~B}$ & 0,26 & 3,54 \\
Foragge $^{\circledR} 60,0 \%$ & 0,77 & $34,5 \mathrm{~A}$ & 0,20 & 3,58 \\
Foragge $^{\circledR} 65,0 \%$ & 0,85 & $28,0 \mathrm{~A}$ & 0,13 & 3,30 \\
\hline MG & $\mathbf{0 , 8 1}$ & $\mathbf{2 5 , 2 1}$ & $\mathbf{0 , 1 8}$ & $\mathbf{3 , 4 7}$ \\
CV & $\mathbf{1 4 , 9 8}$ & $\mathbf{1 2 , 3 6}$ & $\mathbf{2 8 , 3 7}$ & $\mathbf{2 , 4 - 3 , 0}$ \\
\hline VR $^{*}$ & $\mathbf{1 , 2 - 1 , 9}$ & $\mathbf{1 7 - 4 3}$ & $\mathbf{0 - 1 , 9}$ & \\
\hline Tratamento $^{*}$ & $\mathrm{PT}(\mathrm{g} / \mathrm{dL})$ & Triglicerídeo (mg/dL) & Colesterol (mg/dL) & \\
\hline Silagem de milho* & 5,27 & 22,47 & 60,80 & \\
Foragge $^{\circledR} 52,5 \%$ & 5,05 & 15,33 & 54,67 & \\
Foragge $^{\circledR} 60,0 \%$ & 5,47 & 18,77 & 55,5 & \\
Foragge $^{\circledR} 65,0 \%$ & 5,35 & 20,67 & 55,67 & \\
\hline MG $^{*}$ & $\mathbf{5 , 2 8}$ & $\mathbf{1 9 , 3 1}$ & $\mathbf{5 6 , 6 6}$ & $\mathbf{1 3 , 9 5}$ \\
CR $^{*}$ & $\mathbf{6 , 2 5}$ & $\mathbf{8 , 8 1}$ & $\mathbf{5 6 - 7 2}$ &
\end{tabular}

PT = Proteína bruta; MG = média geral; CV= coeficiente de variação; VR = valor de referência; * Kaneko et al., (2008); González (2000); Meyer (2004). *Silagem de milho na forma convencional como tratamento controle. *Silagem de milho na forma convencional como tratamento controle.

Segundo Van Soest (1994), o amido tem alta digestibilidade, entretanto sua extensão de digestão ruminal pode ser influenciada entre outros fatores pelo processamento do grão e nível de ingestão. Neste sentido, o aumento no CMS pelos animais alimentados com volumoso extrusado refletiu em maiores concentrações de ureia sanguínea, obtidas devido a maior disponibilidade de proteína altamente degradável no rúmen após a extrusão. Mertens (2011) e Patton (1994) encontraram níveis de amido na silagem de milho de $27,7 \%$ e 39,4 $\pm 9,5 \%$ respectivamente, valores estes superiores ao volumoso extrusado. 
A creatinina possui estreita relação com a massa muscular que varia de acordo com o grau de exercício realizado pelos animais (Schutte et al., 1981). Os valores observados neste experimento ficaram $67,5 \%$ abaixo do nível mínimo indicado na literatura (Tabela 5). Isto está relacionado à limitação de movimentação por parte dos animais, uma vez que foram mantidos em gaiolas metabólicas e não realizavam atividades rotineiras da espécie, como pastejar, andar, entre outras. Além disso, a creatinina também está ligada às funções renais, o que poderia ter contribuído para um aumento na densidade de urina, associada a baixa ingestão de água (Tabela 3). Porém, vale ressaltar que os animais não passaram por estresse hídrico e suas funções renais não foram alteradas (Tabela 3), uma vez que a densidade de urina se manteve apenas $1,7 \%$ acima do recomendado.

A concentração de ácido úrico indica correlação positiva com a quantidade de proteína microbiana disponível para a digestão intestinal no animal (Giesecke et al., 1994) e capacidade fermentativa do rúmen, estando relacionado com os valores observado para a DMS (Tabela 2). Os valores encontrados no presente estudo mantiveram-se dentro do indicado para espécie ovina (Tabela 5).

A concentração de albumina manteve-se em média $0,47 \mathrm{~g} / \mathrm{dL}$ acima do recomendado (Tabela 5). Este metabólito constituiu as principais proteínas plasmáticas, desempenhando várias funções como: manutenção da pressão osmótica e viscosidade do sangue, transporte de nutrientes, metabólitos, hormônios e produtos de excreção, regulação do $\mathrm{pH}$ sanguíneo e coagulação sanguínea (González, 2006). A albumina também é considerada um indicador mais sensível para determinar o estado nutricional proteico, de modo que valores persistentemente baixos sugerem inadequado consumo proteico. Nesse sentido, podemos concluir que não houve consumo de proteína abaixo do requerido pelas ovelhas no presente estudo.

A quantidade de proteína total indica o estado nutricional proteico do animal e sua redução pode ocasionar falhas hepáticas, renais, intestinais, hemorragias e deficiências nutricionais (González, 2000). Apesar de apresentar valores médios de $12 \%$ abaixo do ideal para a espécie (Tabela 5) não foram observados problemas fisiológicos durante o experimento. Isso indica que não se pode analisar um metabólito isolado do outro, uma vez que os demais relacionados ao estado proteico da dieta mantiveram-se dentro do previsto na literatura, como por exemplo a ureia (Tabela 5), e o CMS esteve acima do recomendado para a espécie (Tabela 2 ).

Os triglicerídeos servem, principalmente, como fonte de energia metabólica celular, acumulando-se no tecido adiposo, de onde são mobilizados em resposta às demandas energéticas do corpo. A concentração sérica dos triglicerídeos está de acordo com as faixas recomendadas para a espécie ovina (Kaneko et al. 2008) (Tabela 5). O colesterol não sofreu influência dos tratamentos $(P>0,05)$ e manteve-se próximo ao valor mínimo recomendado na literatura (Tabela 5). Colesterol e ureia utilizam o mesmo substrato para sua formação, o piruvato. O piruvato dá origem a Acetil CoA e está molécula é responsável pelo enlongamento da cadeia lipídica, enquanto que para a formação da ureia é utilizado o oxalacetato, que por sua vez também é derivado do piruvato (Motta, 2011). Como houve aumento nos níveis de ureia sanguínea, houve redução na formação do colesterol, devido ao possível desvio do substrato piruvato. Além disso, os ingredientes que compunham a dieta continham baixa quantidade de EE (Tabela 1).

Não houve efeito dos tipos de volumosos e do período de coleta a concentração de glicose sanguínea nos animais ( $P \geq 0,05$; Tabela 6$)$. Todos os valores de glicemia mantiveram-se dentro da faixa descrita por Kaneko (2008), de $50-80 \mathrm{ml} \mathrm{dL}^{-1}$. De acordo com o NRC (2007) dietas que elevam a proporção do ácido propiônico na fermentação ruminal podem elevar a produção de glicose no fígado. Diversos estudos relacionam o aumento de ácido propiônico com a inclusão de concentrado nas dietas (Amaral et al. 2009; Lana e Russel, 2001. A presença de amido tanto na silagem de milho quanto no volumoso extrusado contribuiu para esta normalidade, além de proporcionar manutenção da estabilidade energética durante todo o período, evitando possíveis problemas fisiológicos. No ruminante, o amido passa primeiro por fermentação microbiana no rúmen, com consequente produção de células microbianas e ácidos graxos voláteis (AGV), e o que não é transformado sofre, posteriormente, digestão enzimática no intestino delgado com liberação de glicose (Waldo, 1973). A manutenção de níveis estáveis para glicemia ao longo do dia mantém o aporte energético para os animais e evita a ocorrência de distúrbios metabólicos.

\section{Conclusão}

O volumoso extrusado com até 65\% de Uruchloa brizantha em sua composição pode substituir a silagem de milho sem causar transtornos metabólicos para ovinos. Sua utilização promove aumento no consumo de matéria seca pelos animais sem reduzir a digestibilidade do alimento e sem causar efeitos deletérios nos parâmetros bioquímicos e comportamento ingestivo.

\section{Aprovação do Comitê de Ética}

Os dados foram submetidos ao Comitê de Ética na Utilização de Animais obtendo aprovação sob o protocolo 094/17. 
Tabela 6 - Glicemia e curva glicêmica de ovelhas alimentadas com diferentes tipos de volumoso extrusado em comparação à silagem de milho.

\begin{tabular}{cc}
\hline Tratamento & Glicemia (mg/dL) \\
\hline Silagem de milho & 56,76 \\
Foragge $^{\circledR} 52,5 \%$ & 62,64 \\
Foragge $^{\circledR} 60,0 \%$ & 61,96 \\
Foragge $^{\circledR} 65,0 \%$ & 61,48 \\
\hline CV & $\mathbf{1 1 , 1 6}$ \\
MG & $\mathbf{6 0 , 7 1}$ \\
\hline Horário & Glicemia (mg/dL) \\
\hline $08: 00$ & 57,25 \\
$11: 00$ & 62,25 \\
$14: 00$ & 61,30 \\
$17: 00$ & 61,25 \\
$20: 00$ & 61,50 \\
\hline
\end{tabular}

\section{Referências}

Andrigueto, J. M.; Perly, L.; Minardi, I.; Gemael, A.; Flemming, J. S.; Souza, G. A.; Bona Filho, A. 1981. Nutrição animal: as bases e os fundamentos da nutrição animal. 4. Ed. Nobel, São Paulo, Brasil.

Amaral, D. F.; Barbosa, O.R.; Gasparino, E.; Akimoto, L. S.; Lourenço, F. J.; Santello, G. A. 2009. Efeito da suplementação alimentar nas respostas fisiológicas, hormonais e sanguíneas de ovelhas Santa Inês, Ile de France e Texel. Acta Scientiarum Animal Sciences. 31: 403-410. Doi: 10.4025/ actascianimsci.v31i4.4920 Disponível em: file:///C:/Users/Carol/ Downloads/Efeito_da_suplementacao_alimentar_nas_respostas_fi.pdf.

Araújo Filho, J.A. 2006. Aspectos zooecológicos e agropecuários do caprino e do ovino nas regiões semiáridas. Sobral: Embrapa Caprinos. Disponível em: https://ainfo.cnptia.embrapa.br/digital/bitstream/ CNPC/20255/1/doc61.pdf.

Bürger, P.J.; Pereira, J.C.; Queiroz, A.C.; Silva, J.F.C.; Valadares Filho, S.C.; Cecon, P.R. e Casali, A.D.P. 2000.Comportamento ingestivo em bezerros holandeses alimentados com dietas contendo diferentes níveis de concentrado. Revista Brasileira de Zootecnia, 29: 236-242. Doi: https://doi.org/10.1590/S1516-35982000000100031 Disponível em: https://www.scielo.br/pdf/rbz/v29n1/5754.pdf.

Campling, R. C. e Balch, C. C. 1961. Faetors affecting the voluntary intake of food by cows. 1. Preliminary observations of the effect, on the voluntary intake of hay, of the changes in the amount of the reticulo-ruminal contents. British Journal of Nutrition, 16: 523-530. Doi: https://doi.org/10.1079/BJN19610065 Disponível em: https:// www.cambridge.org/core/services/aop-cambridge-core/content/ view/0BF2673C81B4EBB5CF33EBB01454526F/S000711456100066Xa. pdf/factors_affecting_the_voluntary_intake_of_food_by_cows.pdf.

Fagundes, M. H. R. 2005. Efeito do sistema de fornecimento de alimentos e processamento do concentrado sobre a digestibilidade da dieta e resposta glicêmica plasmática, em equinos. Pirassununga: Faculdade de Medicina Veterinária e Zootecnia, Universidade de São Paulo, 90f. Dissertação Mestrado. Disponível em: https://www.teses. usp.br/teses/disponiveis/10/10135/tde-10042007-162440/publico/ MarcoHFagundes.pdf.
Fischer, H.; Wagenbach, D.; Kipfstuhl, J. 1998. Sulfate and nitrate firn concentrations on the Greenland ice sheet: 1 . Large-scale geographical deposition changes. Journal of Geophysical Research. 103: 927-934. Doi: https://doi.org/10.1029/98JD01885 Disponível em: https://agupubs. onlinelibrary.wiley.com/doi/epdf/10.1029/98JD01885.

Forbes, J.M. 1968. The water intake of ewes. British Journal Nutrition, 22:.33-43. Disponível em: https://www.researchgate.net/profile/ John Forbes3/publication/17541189 The water intake of ewes/ links/5660369d08ae4988a7bf093d.pdf.

Giesecke, D.; Ehrentreich, L.; Stangassinger, M. 1994. Mammary and renal excretion of purine metabolites in relation to energy intake and milk yield in dairy cows. Jounal of Dairy Science, 77:2376-2381. Doi: doi: 10.3168/jds.S0022-0302(94)77180-0 Disponível em: https:// pubmed.ncbi.nlm.nih.gov/7962859/.

Gomes S. P.; Borges I.; Borges A. L. C. C.; Macedo Junior G. L.; Campos W. E.; Brito T. S. 2012.Tamanho de partícula do volumoso e freqüência de alimentação sobre o metabolismo energético e protéico em ovinos, considerando dietas com elevada participação de concentrado. Revista Brasileira de Saúde e Produção Animal, 13: 732-744. Doi: https://doi. org/10.1590/S1519-99402012000300013 Disponível em: https://www. scielo.br/scielo.php?pid=S1519-99402012000300013\&script $=$ sci abstract\&tlng $=$ pt.

González, F.H.D. 2000. Uso do perfil metabólico para determinar o status nutricional em gado de corte. P.32-72. In: González, F.H.D.; Barcellos, J.O.; Patiño, H.O.; Ribeiro, L.A. O Perfil metabólico em uso em nutrição e doenças nutricionais. UFRGS, Porto Alegre, Brasil. Disponível em: https://lume.ufrgs.br/bitstream/handle/10183/26687/000299715. pdf? sequence $=1$ \&isAllowed $=\mathrm{y}$.

González, F.H.D.; Sheffer, J.F.S. 2002. Perfil sanguíneo: ferramenta de análise clínica, metabólica e nutricional. Anais do 29ํㅡㄹ Congresso de Medicina Veterinária, Gramado, Brasil. Disponível em: https://www. lume.ufrgs.br/bitstream/handle/10183/13177/000386508.pdf. 
Parâmetros nutricionais e bioquímicos de ovinos consumindo volumoso extrusado com diferentes teores de Uruchloa brizantha em comparação a silagem de milho tradicional

González, F.H.D.; Silva, S.C. 2006. Introdução à bioquímica clínica veterinária. Porto Alegre: Gráfica da Universidade Federal do Rio Grande do Sul.

Hendrix, C.M. 2005. Procedimentos laboratoriais para técnicos veterinários. 4. ed. Rocca, São Paulo, Brasil.

Kaneko, J.J; Harvey, J.W; Bruss, M.L. 2008. Clinical Biochemistry of Domestic Animals. 6. ed. Academic Press, San Diego.

Kruskal, W.H.; Wallis, W.A. 1952. Use of ranks em one-criterion variance analysis. Jounal American Statistical Association. 47: 583-621.

Lana, R. P.; Russell, J. B. 2001. Efeitos da Monensina sobre a Fermentação e Sensibilidade de Bactérias Ruminais de Bovinos sob Dietas Ricas em Volumoso ou Concentrado. Revista Brasileira de Zootecnia. 30: 254 260. Disponível em: https://www.scielo.br/pdf/rbz/v30n1/5461.pdf.

Maynard, L.A.; Looslo, B.S.; Hintz, H.F.; Warner, R.G. Nutrição animal. 3.ed. Freitas Bastos, Rio de Janeiro, RJ, Brasil.

Meyer, D.J.; Harvey, J.W. 2004. Veterinary laboratory medicine: interpretation \& diagnostic. 2.ed. Sauders, Philadelphia.

Mertens, D.R.; Broderick, G.A.; Simons, R. 1994. Efficacy of carbohydrate sources for improving utilization of $\mathrm{N}$ in alfalfa silage. Journal of Dairy Science, 77: 240.

Motta, V. T. 2011. Bioquímica, Editora Medbook. 2.ed, Medbook.

National Research Council - NRC. 2007. Nutrient requirements of small ruminants: sheep, goats, cervids, and new world camelids. National Academy Press, Washington, USA.

Patton, R.S. 1994. Complexities of soluble carboydrate metabolism in ruminants. Feedstuffs, 66: 1624-1633.

Polli, V. A.; Restle, J.; Senna, D. B.; Rosa, C. E.; Aguirre, L. F.; DA Silva, J. 1996. Aspectos relativos à ruminação de bovinos e bubalinos em regime de confinamento. Revista Brasileira de Zootecnia, 24: 987-993.
Reece, W. O. 2006. Função Renal nos Mamíferos. p. 68-96. In: Reece.; W. O. Dukes., 12.eds. Fisiologia dos animais domésticos. Guanabara Koogan, Rio de Janeiro, RJ, Brasil.

Rodrigues, R..C. 2010. Métodos de análises bromatológicas de alimentos: métodos físicos, químicos e bromatológicos. Pelotas: Embrapa Clima Temperado. Disponível em: https://ainfo.cnptia.embrapa.br/digital/ bitstream/item/40059/1/documento-306.pdf.

Schutte, J.E.; Longhurst, J.C.; Gaffney, F.A.; Bastian, B.C.; Blomqvist, C.G. 1981. Total plasma creatinine: an accurate measure of total striated muscle mass. Journal of Applied Physiology, 51:762-766. Doi: https:// doi.org/10.1152/jappl.1981.51.3.762 Disponível em: https://journals. physiology.org/doi/abs/10.1152/jappl.1981.51.3.762.

Senger, C.C.D.; Muhlbach, P.R.F.; Sánchez, L.M.B.; Peres Netto, D.; De Lima, L.D. 2005. Composição química e disgetibilidade "in vitro" de silagens de milho com distintos teores de umidade e níveis de compactação. Revista Ciência Rural, 35: 1393-1399. Dosponível em: https://pesquisa.bvsalud.org/portal/resource/pt/lil-417681.

Van Cleef, E. H. C. B.; Ezequiel, J. M. B.; D’Aurea, A. P.; Fávaro, V. R.; Sancanari, J. B. D. 2010. Crude glycerin in diets for feedlot Nellore cattle. Revista Brasileira de Zootecnia, 43: 86-91.

Van Soest, P.J. Nutritional ecology of the ruminant. 2.ed. Ithaca: Cornell University Press, 476p, 1994. Doi: https://doi.org/10.1590/S151635982014000200006 Disponível em: https://www.scielo.br/scielo. php?script $=$ sci_arttext\&pid $=$ S1516-35982014000200006.

Waldo, D.R. 1973. Extent and partition of cereal grain starch digestion in ruminants. Journal of Animal Science, 37:1062-1074. Doi: https://doi. org/10.2527/jas1973.3741062x Disponível em : https://academic.oup. com/jas/article-abstract/37/4/1062/4667577? redirectedFrom=fulltext.

Wittwer, F. 2000. Diagnóstico dos desequilíbrios metabólicos de energia em rebanhos bovinos. P. 9-22. In: González, F.H.D. O perfil metabólico em uso em nutrição e doenças nutricionais. Gráfica da Universidade Federal do Rio Grande do Sul, Porto Alegre, Brasil. 